\title{
Corrigendum: Perceived Impact as the Underpinning Mechanism of the End-Spurt and U-Shape Pacing Patterns
}

\author{
Aviv Emanuel* \\ School of Psychological Sciences, Tel Aviv University, Tel Aviv, Israel
}

Keywords: motivation, pacing, end-spurt, U-shape, effort

\section{A Corrigendum on}

Perceived Impact as the Underpinning Mechanism of the End-Spurt and U-Shape Pacing Patterns

by Emanuel, A. (2019). Front. Psychol. 10:1082. doi: 10.3389/fpsyg.2019.01082

In the original article, there was an error. In equations (1) and (2), the nominator should have been "1." In addition, the text should have clearly indicated this fact.

A correction has been made to the Formulation of the Perceived Impact Mechanism section, paragraph one:

"To illustrate, perceived impact of a step in goal-pursuit can be stated formally by a simple function. For example, let a step in goal-progress be equal to one, $s$ be a series of numbers in an increasing order, representing the index of each step (e.g., $s=1,2,3,4,5,6,7)$, and $P I_{s_{i}}$ a number between 0 to 100 , representing the percent of perceived impact of the current step $s_{i}$ (e.g., $P I_{s_{i}}=$ $50 \%$; representing the impact of the current step out of the maximum possible impact a step can have on goal-progress). Accordingly, $\min (s)$ and $\max (s)$ are the smallest and highest values in $s$, which represent the starting- and ending-points, respectively. According to the small area principle, people use the nearest reference point, and tend to switch between the beginning and ending points in the middle of the task. Therefore, if $s_{i}<\frac{\max (s)}{2}$, then:

$$
P I_{s_{i}}=\frac{1}{\min (s)+s_{i}}
$$

Else:

$$
P I_{s_{i}}=\frac{1}{\max (s)-s_{i}}
$$

Received: 11 June 2019 Accepted: 25 June 2019

Published: 09 July 2019

Citation:

Emanuel A (2019) Corrigendum: Perceived Impact as the Underpinning Mechanism of the End-Spurt and

U-Shape Pacing Patterns.

Front. Psychol. 10:1597.

doi: 10.3389/fpsyg.2019.01597

The author apologizes for this error and state that this does not change the scientific conclusions of the article in any way. The original article has been updated.

Copyright (C) 2019 Emanuel. This is an open-access article distributed under the terms of the Creative Commons Attribution License (CC BY). The use, distribution or reproduction in other forums is permitted, provided the original author(s) and the copyright owner(s) are credited and that the original publication in this journal is cited, in accordance with accepted academic practice. No use, distribution or reproduction is permitted which does not comply with these terms. 